\title{
Multisubband Plasmons in Doped ZnO Quantum Wells
}

\author{
Miguel Montes Bajo, ${ }^{1}$ Julen Tamayo-Arriola, ${ }^{1}$ Maxime Hugues, ${ }^{2}$ Jose M. Ulloa, ${ }^{1}$ Nolwenn Le Biavan, ${ }^{2}$ \\ Romain Peretti, ${ }^{3}$ Francois H. Julien, ${ }^{4}$ Jerome Faist,${ }^{3}$ Jean-Michel Chauveau, ${ }^{2}$ and Adrian Hierro ${ }^{1, *}$ \\ ${ }^{1}$ ISOM, Universidad Politécnica de Madrid, 28040 Madrid, Spain \\ ${ }^{2}$ Université Côte d'Azur, CNRS, CRHEA, 06560 Valbonne, France \\ ${ }^{3}$ Institute for Quantum Electronics, ETH Zürich, Switzerland \\ ${ }^{4}$ Centre de Nanosciences et de Nanotechnologies, CNRS, University Paris-Sud, University Paris-Saclay, \\ C2N-Orsay, 91405 Orsay Cedex, France
}

(Received 1 March 2018; revised manuscript received 21 May 2018; published 6 August 2018)

\begin{abstract}
Intersubband (ISB) transitions are of high significance for light-emitting and light-detecting devices in the infrared and, when involving large electron densities, for plasmonics and strong light-matter coupling physics. Here it is observed that the simultaneously occurring fundamental and excited-state ISB transitions in highly-doped, $m$-plane $\mathrm{ZnO} / \mathrm{Mg}_{x} \mathrm{Zn}_{1-x} \mathrm{O}$ multiple quantum wells, couple into a single collective oscillation: the multisubband plasmon (MSP). With 2D electron densities up to $4 \times 10^{13} \mathrm{~cm}^{-2}$, an outstanding regime is reached in which the observed MSP frequency is three times larger than that of the fundamental ISB transition as a result of depolarization. This effect is analyzed using a dielectric tensor for $\mathrm{ZnO}$ including the interaction of the light with the lattice, the in-plane free electrons, and the off-plane MSP. The impact of the broadening of the MSP and its interaction with phonons is discussed. The results presented here show the potential of $\mathrm{ZnO} / \mathrm{Mg}_{x} \mathrm{Zn}_{1-x} \mathrm{O}$ for infrared optoelectronic applications, which can be extended to the $\mathrm{THz}$ range with appropriate design of the quantum wells.
\end{abstract}

DOI: 10.1103/PhysRevApplied.10.024005

\section{INTRODUCTION}

Oxides are semiconductors where very high crystallinity control over the heterointerfaces has been achieved recently, allowing phenomena that arise from the presence of a high-mobility two-dimensional electron gas (2DEG) $[1,2]$ as well as from the strong correlation between electrons to be explored [3]. Of particular interest is the $\mathrm{ZnO} / \mathrm{Mg}_{x} \mathrm{Zn}_{1-x} \mathrm{O}$ material system, where the quantum Hall effect (QHE) has been observed in heterostructures grown by molecular beam epitaxy (MBE) with electron densities up to $3.7 \times 10^{12} \mathrm{~cm}^{-2}$ [1]. More recently, the observation of the fractional QHE has also been reported in thin layers, where electrons are highly confined and feature mobilities exceeding $1 \times 10^{6} \mathrm{~cm}^{2} \mathrm{~V}^{-1} \mathrm{~s}^{-1}[3,4]$, and indeed the rare even-denominator fractional quantum Hall states have been identified [5]. In contrast to the QHE, the fractional QHE can only be explained as a result of the presence of a strong correlation between charge carriers driven by a Coulomb interaction. This strong correlation can be highly

\footnotetext{
*adrian.hierro@upm.es
}

Published by the American Physical Society under the terms of the Creative Commons Attribution 4.0 International license. Further distribution of this work must maintain attribution to the author(s) and the published article's title, journal citation, and DOI. enhanced if, as in the $\mathrm{ZnO} / \mathrm{Mg}_{x} \mathrm{Zn}_{1-x} \mathrm{O}$ system, the electrons feature a very large effective mass $\left(0.24 m_{0}\right.$ in $\mathrm{ZnO}$ [6], e.g., much larger than that of GaAs, $0.0637 m_{0}$ [7], where $m_{0}$ is the free-electron mass).

The large electron effective mass in the $\mathrm{ZnO} / \mathrm{Mg}_{x} \mathrm{Zn}_{1-x} \mathrm{O}$ system is further increased as a result of the high ionicity of these polar oxides, which produces a large coupling between electrons and phonons. This strong interaction can be described by a quasiparticle, the polaron [8], with an effective mass $m^{*} \approx 0.28-0.32 m_{0}$ in $\mathrm{ZnO}[1,9]$. This large electron effective mass implies that the curvature of the subbands in $\mathrm{ZnO}$-based quantum wells (QWs) is so small that the QWs can be heavily doped while keeping the electrons within a narrow energy range. As a consequence, it maintains a large electron population difference between the first and second confined levels in the QW, $\Delta n_{12}$. Thus, while $\Delta n_{12}$ can typically be up to approximately $10^{12} \mathrm{~cm}^{-2}$ in GaAs, it can reach values above $10^{13}$ $\mathrm{cm}^{-2}$ in $\mathrm{ZnO}$. The unique combination of large effective mass and very high 2D electron carrier concentrations in $\mathrm{ZnO}$ opens a path to explore phenomena that scale with electron density in the QW, such as the depolarization shift in intersubband (ISB) plasmons [10,11], and phenomena occurring in the ultrastrong ISB-phonon coupling regime, i.e., ISB polarons [12].

Here, we explore ISB transitions in Ga-doped, $m$ plane $\mathrm{ZnO} / \mathrm{Mg}_{x} \mathrm{Zn}_{1-x} \mathrm{O}$ multiple quantum wells (MQW) 
featuring very high $2 \mathrm{D}$ electron concentrations. $\mathrm{ZnO}$ is a material with great potential for ISB transition-based detectors and emitters from the midinfrared to the $\mathrm{THz}$, since it offers several singular characteristics that are not present in the commonly used GaAs-based systems. ISB transition-based absorption and detection have already been observed in $\mathrm{ZnO}$ quantum wells [13-15], and the development of quantum cascade lasers in the $\mathrm{THz}$ range would benefit from the large phonon energy of $\mathrm{ZnO}$ (approximately $72 \mathrm{meV}$ ) [16].

The $\mathrm{ZnO} / \mathrm{Mg}_{x} \mathrm{Zn}_{1-x} \mathrm{O}$ material system is also chosen in this work because of the availability of nonpolar, native $\mathrm{ZnO}$ substrates. To date, ISB transitions in wurtzite wideband-gap semiconductors, including the $\mathrm{GaN}$ and $\mathrm{ZnO}$ material systems, have mainly been observed in polar QWs grown along the $c$ axis (mostly on foreign substrates, e.g., sapphire or $\mathrm{Si}$ ), where the quantum confined Stark effect (QCSE) takes place $[14,15,17]$. Growing the QW structures on a nonpolar orientation (i.e., with the $c$ axis parallel to the interface between layers) can suppress the QCSE [18] and should facilitate the ISB transitions due to the absence of internal electric fields that have a detrimental impact on the oscillator strength of the transitions [13]. Growing the QW structures on native substrates should also drastically improve the ISB transition-based devices by reducing the defect density [19] and the residual electron concentration of the films, which is as low as (1-5) $\times 10^{14} \mathrm{~cm}^{-3}$ in the homoepitaxial $m-\mathrm{ZnO} / \mathrm{Mg}_{x} \mathrm{Zn}_{1-x} \mathrm{O}$ QWs used in this report [20]. The combination of extremely high doping capability of up to $1 \times 10^{21} \mathrm{~cm}^{-3}$ [21] and low residual electron concentration of $\mathrm{ZnO}$ and $\mathrm{Mg}_{x} \mathrm{Zn}_{1-x} \mathrm{O}$ provides an unprecedented span of controllable $2 \mathrm{D}$ electron concentrations.

\section{METHODS}

\section{A. Multiple-quantum-well structures}

The m-plane multiple QW structures presented here, grown by $\mathrm{MBE}$ on $m$-plane $\mathrm{ZnO}$ substrates, are shown schematically in Fig. 1(a). Samples $A$ and $B$ feature 3.7-nm-wide QWs and are Ga-doped $1 \times 10^{19}$ and $2 \times 10^{19} \mathrm{~cm}^{-3}$, respectively. Sample $C$ has 4 -nm-wide QWs doped $6 \times 10^{19} \mathrm{~cm}^{-3}$ in the central $2 \mathrm{~nm}$ of the QW only. An additional undoped sample with nominally identical structure as sample $C$ is also used as a reference in the reflectance studies. Finally, sample $D$ features 3.9-nm-wide QWs doped $1 \times 10^{20} \mathrm{~cm}^{-3}$.

Figure 1(b) shows an example of the potential profile of one of the QWs presented here as well as the Fermi energy for all samples. The values of $\omega_{12}$ and $\omega_{23}$ in the QWs are calculated from the self-consistent solution of the Schrödinger and Poisson equations. Both the barrier and QW electron masses are taken to be the $\mathrm{ZnO}$ polaron mass $0.28 m_{0}$ [9]. The band offset, $\Delta E_{c}$, is taken to be $\Delta E_{c}=0.675 E_{g}$, since the reported values range from 0.65 (a)

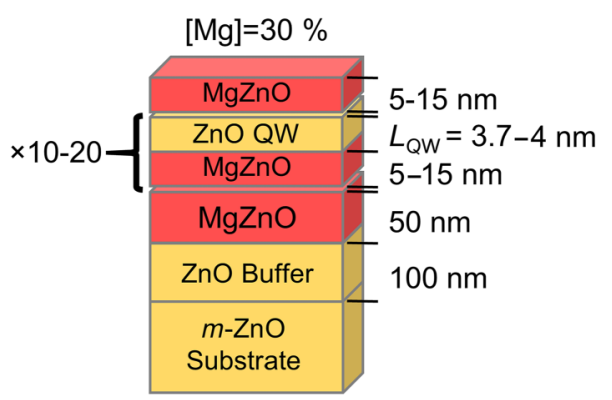

\begin{tabular}{|c|c|c|}
\hline Sample & $\begin{array}{c}\text { QW width } \\
(\mathrm{nm})\end{array}$ & $\begin{array}{c}\text { Ga doping } \\
\left(\mathrm{cm}^{-3}\right)\end{array}$ \\
\hline A & 3.7 & $1 \times 10^{19}$ \\
\hline B & 3.7 & $2 \times 10^{19}$ \\
\hline C & 4 & $\begin{array}{c}6 \times 10^{19} \\
(1 / 2 \mathrm{QW})\end{array}$ \\
\hline D & 3.9 & $1 \times 10^{20}$ \\
\hline UID ref & 4 & UID \\
\hline
\end{tabular}

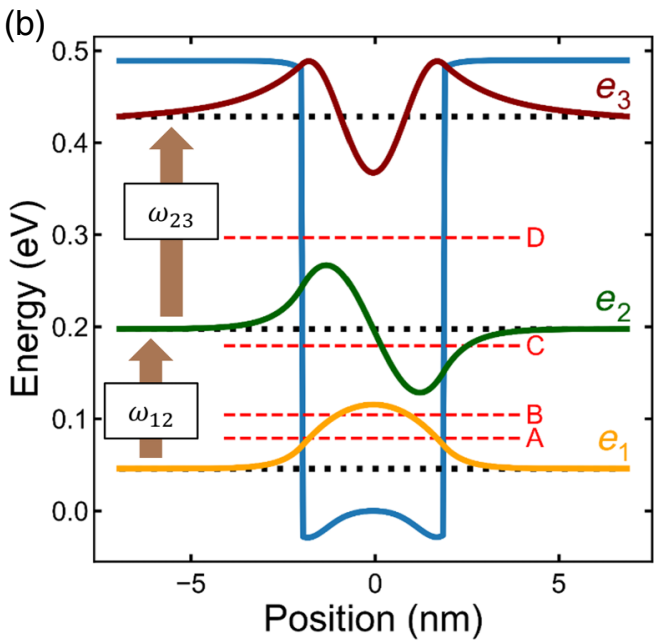

FIG. 1. Details on the samples in this work. (a) Schematic of the multiple quantum well structures presented. The table shows the different doping levels and schemes employed. (b) Potential profile of one of the QWs in sample $D$ calculated from the selfconsistent solution of the Schrödinger and Poisson equations. Wave functions are offset to match their respective energy levels. Red lines indicate the Fermi energy for all samples. The allowed ISB transitions are also indicated.

to $0.70 E_{g}[14,15,22]$. The $\mathrm{Mg}_{x} \mathrm{Zn}_{1-x} \mathrm{O}$ band gap is calculated, as in Neumann et al. [23], using $25 \mathrm{meV}$ per $\%$ of $\mathrm{Mg}$ at $30 \% \mathrm{Mg}$. With this QW model, the oscillator strength of the $e_{1}-e_{2}$ transition, $f_{12}$, is calculated to be between 0.97 and 0.98 , and that of the $e_{2}-e_{3}$ transition, $f_{23}$, between 1.92 and 1.97. The resulting electronic level structure is similar in all the structures studied here: three conductionband levels confined in the QWs with an energy difference between the first two, $E_{12}=\hbar \omega_{12}$, ranging from 1220 to 
$1350 \mathrm{~cm}^{-1}$ (152 to $167 \mathrm{meV}$ ) and an energy difference between the second and third levels, $E_{23}=\hbar \omega_{23}$, ranging from 1810 to $1910 \mathrm{~cm}^{-1}$ (224 to $237 \mathrm{meV}$ ).

\section{B. Transmittance and reflectance spectroscopy}

Slabs 3-mm wide and 6-mm long are cut from the asgrown samples, with the short edges oriented parallel to the $c$ axis. Multipass waveguides are prepared bevelling the short edges at $45^{\circ}$ and polishing them mechanically to optical quality with diamond compounds. The back side of the waveguides is polished to optical quality, too. The transmittance is measured at room temperature in a Fourier transform IR (FTIR) spectrometer using a KRS-5, holographic wire-grid polarizer to select the polarization of the incident light. The samples are set in a specially designed blind that allows light only through the bevelled edges of the waveguide.

Reflectance measurements are performed at room temperature on the as-grown samples at $45^{\circ}$ and $75^{\circ}$ angle of incidence of the light in the same FTIR spectrometer. Since the $c$ axis lies in plane for $m-\mathrm{ZnO}$, in $p$-polarization transmittance and in all reflectance measurements we place the $c$ axis perpendicular to the electric field [24], such that the material properties in plane and out of plane are isotropic (except those that arise from the ISB transition) and correspond to the $\boldsymbol{E} \perp c$ orientation.

\section{Reflectance model}

The reflectance spectra are modeled using a semiclassical dielectric function model for each of the constituent layers. For the $\mathrm{Mg}_{x} \mathrm{Zn}_{1-x} \mathrm{O}$ layers, the dielectric function is isotropic (i.e., equal for in plane and off plane of the $\mathrm{QW}$ ) and includes only the terms accounting for the interaction between the light and the lattice,

$$
\varepsilon_{\mathrm{Mg}_{x} \mathrm{Zn}_{1-x} \mathrm{O}}=\varepsilon_{\infty}^{\mathrm{Mg}_{x} \mathrm{Zn}_{1-x} \mathrm{O}} \prod_{k=1}^{2} \frac{\omega_{\mathrm{LO}, k}^{2}-\omega^{2}-i \gamma_{\mathrm{LO}, k} \omega}{\omega_{\mathrm{TO}, k}^{2}-\omega^{2}-i \gamma_{\mathrm{TO}, k} \omega},
$$

where $k=1,2$ stands for the two phonon modes of nonpolar $\mathrm{Mg}_{x} \mathrm{Zn}_{1-x} \mathrm{O}$ for $\boldsymbol{E} \perp c$, as in Ref. [25]. $\varepsilon_{\infty}^{\mathrm{Mg}_{x} \mathrm{Zn}_{1-x} \mathrm{O}}$ is the high-frequency dielectric constant, $\omega_{\mathrm{LO}, k}$ and $\omega_{\mathrm{TO}, k}$ are the $\mathrm{LO}$ and TO phonon frequencies of the $k$ mode, and $\gamma_{\mathrm{LO}, k}$ and $\gamma_{\mathrm{TO}, k}$ are the LO and TO phonon broadenings of the $k$ mode (see [24] for more details on these figures).

In the case of $\mathrm{ZnO}$, the dielectric function is anisotropic, having a different form in the direction parallel to the QW

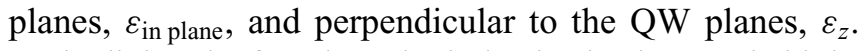
Both dielectric functions include the background, highfrequency dielectric constant, $\varepsilon_{\infty}^{\mathrm{ZnO}}$, and a harmonic oscillator term analogous to that in Eq. (1) to account for the light-phonon interaction [first term in Eqs. (2a) and (2b)]. However, $\varepsilon_{z}$ features an additional harmonic oscillator term to account for the interaction of the light with the
ISB transitions [second term in Eq. (2a), where the sum in $j$ extends to the first two allowed trasitions: $e_{1} \rightarrow e_{2}$ and $\left.e_{2} \rightarrow e_{3}\right]$, and $\varepsilon_{\text {in plane }}$ a Drude model term for the in-plane interaction with the electrons [second term in Eq. (2b)] $[12,26,27]$ :

$$
\begin{aligned}
& \varepsilon_{z}(\omega)=\varepsilon_{\infty}^{\mathrm{ZnO}} \frac{\omega_{\mathrm{LO}}^{2}-\omega^{2}-i \omega \gamma_{\mathrm{LO}}}{\omega_{\mathrm{TO}}^{2}-\omega^{2}-i \omega \gamma_{\mathrm{TO}}}+\sum_{j} \frac{\omega_{p, j}^{2}}{\omega_{j}^{2}-\omega^{2}-i \omega \gamma_{j}}, \\
& \varepsilon_{\text {in plane }}(\omega)=\varepsilon_{\infty}^{\mathrm{ZnO}} \frac{\omega_{\mathrm{LO}}^{2}-\omega^{2}-i \omega \gamma_{\mathrm{LO}}}{\omega_{\mathrm{TO}}^{2}-\omega^{2}-i \omega \gamma_{\mathrm{TO}}}-\frac{\omega_{p}^{2}}{\omega^{2}+i \omega \gamma_{\text {in plane }}^{p}} .
\end{aligned}
$$

Here $\gamma_{j}$ is a phenomenological ISB transition broadening factor we take to be equal to the FWHM of the experimental ISB transition as extracted from the $p: s$ polarization absorbance spectra ratio and $\gamma_{\text {in plane }}^{p}$ stands for the broadening of the plasma resonance in the in-plane direction. $\omega_{p}$ is the plasma frequency of the in-plane light-electron interaction, defined as $\omega_{p}^{2}=n_{3 \mathrm{D}} e^{2} / m^{*} \varepsilon_{0}$, with $n_{3 \mathrm{D}}$ the three-dimensional electron concentration in the $\mathrm{QW}, e$ the electron charge, and $\varepsilon_{0}$ the permittivity of vacuum.

Some of the samples presented here feature doping levels so high it is expected that the transitions between excited levels in the QWs will have a significant impact. In this case, dipole-dipole Coulomb interaction couples the optically active transitions resulting in the appearance of a unique collective mode: a multisubband plasmon (MSP) [28]. This MSP can be thought of as an ISB transition in a QW with only two (virtual) confined levels separated by a frequency $\Omega_{\mathrm{ISBT}}$. The term of the dielectric function corresponding to this mode can be expressed as a single harmonic oscillator as

$$
\frac{\Omega_{p}^{2}}{\Omega_{\mathrm{MSP}}^{2}-\omega^{2}-i \omega \Gamma_{\mathrm{MSP}}}=\sum_{j} \frac{\omega_{p, j}^{2}}{\omega_{j}^{2}-\omega^{2}-i \omega \gamma_{j}},
$$

with an effective plasma frequency defined as a function of the plasma frequencies of the individual ISB transitions, $\omega_{p, j}$, as

$$
\Omega_{p}^{2}=\sum_{j} \omega_{p, j}^{2}=\sum_{j} \frac{f_{j} \Delta n_{2 \mathrm{D}, j} e^{2}}{m^{*} L_{\mathrm{eff}, j} \varepsilon_{0}}
$$

Here, $\Delta n_{2 \mathrm{D}, j}$ is the difference in the two-dimensional electron density between the $j$ and $j+1$ levels. $L_{\text {eff } j}$ is the effective width over which the ISB transitions take place, defined as

$$
L_{\mathrm{eff}, j}=\frac{\hbar}{2 S_{j, j+1} m^{*} \omega_{j}}
$$


where $\omega_{j}$ are the frequencies of the $e_{j}-e_{j+1}$ ISB transitions,

$$
\begin{aligned}
S_{j, j+1}= & \int_{0}^{\infty} \Psi_{j}(z) \Psi_{j+1}(z) \\
& \int_{0}^{z} \int_{0}^{z^{\prime}} \Psi_{j}\left(z^{\prime \prime}\right) \Psi_{j+1}\left(z^{\prime \prime}\right) d z^{\prime \prime} d z^{\prime} d z
\end{aligned}
$$

and $\Psi_{j}(z)$ and $\Psi_{j+1}(z)$ are the electron wave functions corresponding to the levels involved in the $e_{j}-e_{j+1}$ transition $[10,11] . \Omega_{\mathrm{MSP}}$ is in turn the harmonic mean of the ISB transitions weighted by their respective plasma frequencies,

$$
\Omega_{\mathrm{MSP}}^{2}=\frac{\sum_{i} \omega_{p, j}^{2}}{\sum_{j} \omega_{p, j}^{2} / \omega_{j}^{2}} .
$$

The reflectance spectra are modeled with the dielectric function approach described above in a transfer matrix formalism (see Ref. [29] and references cited therein) taking into account the whole multilayer structure. The substrate is considered as a semiinfinite medium to account for the light dispersion at the unpolished back side. The fact that the MSP takes place in a width $L_{\text {eff }}^{\mathrm{MSP}}$ different from the QW width, $L_{\mathrm{QW}}$, is taken into account in the model by setting the central $L_{\mathrm{eff}}^{\mathrm{MSP}}$ of each QW as the doped material and the rest of the QW as the undoped material. $L_{\text {eff }}^{\mathrm{MSP}}$ is extracted from Eq. (4) defining $\Omega_{p}^{2}=f_{\mathrm{MSP}} \Delta n_{2 \mathrm{D}} e^{2} / m^{*} L_{\text {eff }}^{\mathrm{MSP}} \varepsilon_{0}$ by analogy to the individual $\omega_{p, j}$ and making (a) $f_{\mathrm{MSP}} \approx 1$ because the MSP model assumes there are only two (virtual) electronic levels in the QW, and (b) $\Delta n_{2 \mathrm{D}} \approx$ $n_{2 \mathrm{D}}$, yielding $1 / L_{\mathrm{eff}}^{\mathrm{MSP}}=\sum_{j}\left(f_{j} \Delta n_{2 \mathrm{D}, j} / L_{\mathrm{eff}, j}\right) / n_{2 \mathrm{D}}$. Since the MSP treatment reduces to that of a single ISB transition for lowly doped samples, it is used for all samples in this study.

The $\mathrm{Mg}_{x} \mathrm{Zn}_{1-x} \mathrm{O}$ in the barrier layers, the undoped $\mathrm{ZnO}$ in the substrate, and the fraction of the QW outside $L_{\text {eff }}^{\mathrm{MSP}}$ are homogeneous and isotropic [i.e., the same off-plane and in-plane dielectric function, since $\omega_{p}=\Omega_{p}=0$, and Eqs. (2a) and (2b) are then identical]. In contrast, in the doped $\mathrm{ZnO}$ in the QW $\omega_{p} \neq 0$ and $\Omega_{p} \neq 0$, and it has to be described with both the off-plane [Eq. (2a)] and in-plane [Eq. (2b)] dielectric functions.

Setting $\omega_{p}=\Omega_{p}=0$ for $\mathrm{ZnO}$ in the QWs of the undoped reference sample, the frequencies and broadenings of the light-phonon interaction terms of both $\mathrm{ZnO}$ and $\mathrm{Mg}_{x} \mathrm{Zn}_{1-x} \mathrm{O}$ are extracted by fitting the model to the experimental reflectance spectra as shown in Figs. 2(a) and 2(b), yielding values in agreement with the literature $[16,25]$. The values thus obtained [24] are then used as a reference for the analysis of the doped samples and $\omega_{p}$ is extracted for all the samples presented here by fitting their reflectance spectra (Fig. 3). The resulting $n_{3 \mathrm{D}}$ are then used to calculate the individual $\Delta n_{2 \mathrm{D}, j}$, which were subsequently fed into Eq. (4) to calculate $\Omega_{p}$. As discussed in
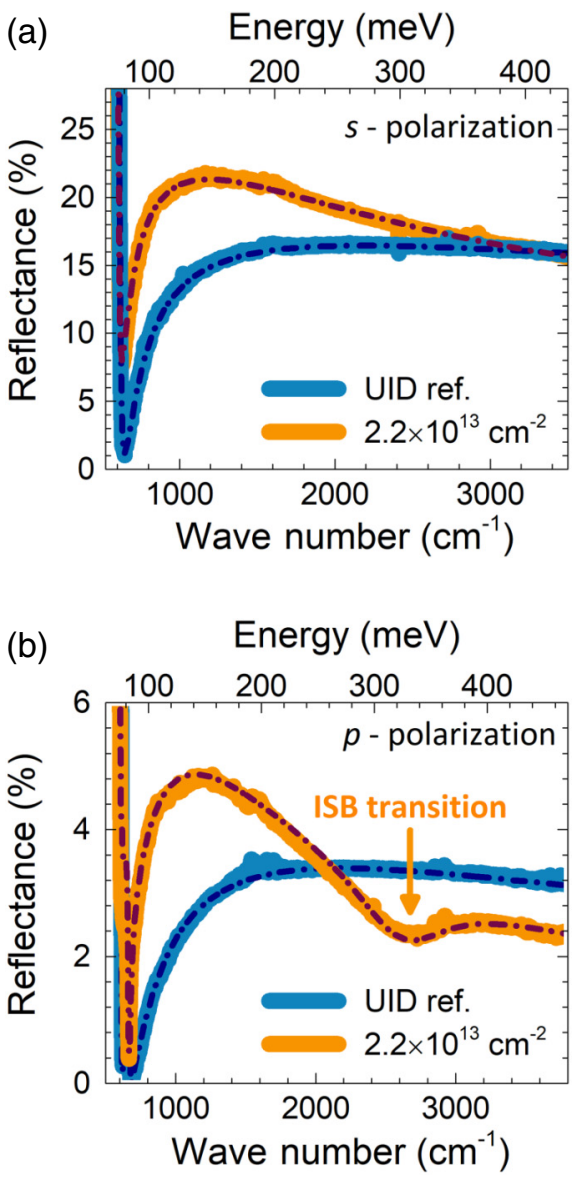

FIG. 2. IR reflectance measurements and fits. (a) Sample $C$ and its equivalent undoped reference under $s$ polarization of the incident light. (b) Same as (a) but under $p$ polarization. The best fit for each spectra is overlaid on the experimental data in all the figures (dashed lines). Legends show the values of $n_{2 \mathrm{D}}$ extracted from reflectance experiments in units of $\mathrm{cm}^{-2}$ (see Sec. II and Ref. [24]).

[24], the detection limit for the QW 2D electron concentration is approximately $(0.3-0.6) \times 10^{12} \mathrm{~cm}^{-2}$, and therefore the technique is not sensitive to the residual electron concentration in these films $\left[(\sim 0.4-2) \times 10^{8} \mathrm{~cm}^{-2}\right]$.

\section{Calculation of the dispersion relation from the absorption spectra}

The dispersion relation of the MSP is calculated from the simulated absorption spectra, $\alpha(\omega)$, as a function of the $2 \mathrm{D}$ electron concentration in the QWs. The absorption spectra are in turn calculated from the off-plane dielectric function of the $\mathrm{ZnO}$ QWs [Eq. (2a)] as [29,30]

$$
\alpha(\omega)=-\operatorname{Im}\left[\frac{\varepsilon_{\infty}}{\varepsilon_{z}}\right] n_{b} \frac{\omega}{c} \frac{\sin ^{2} \theta}{\cos \theta},
$$

where $n_{b}$ is the background, frequency-independent refractive index, $c$ is the speed of light in vacuum, and $\theta$ is the 

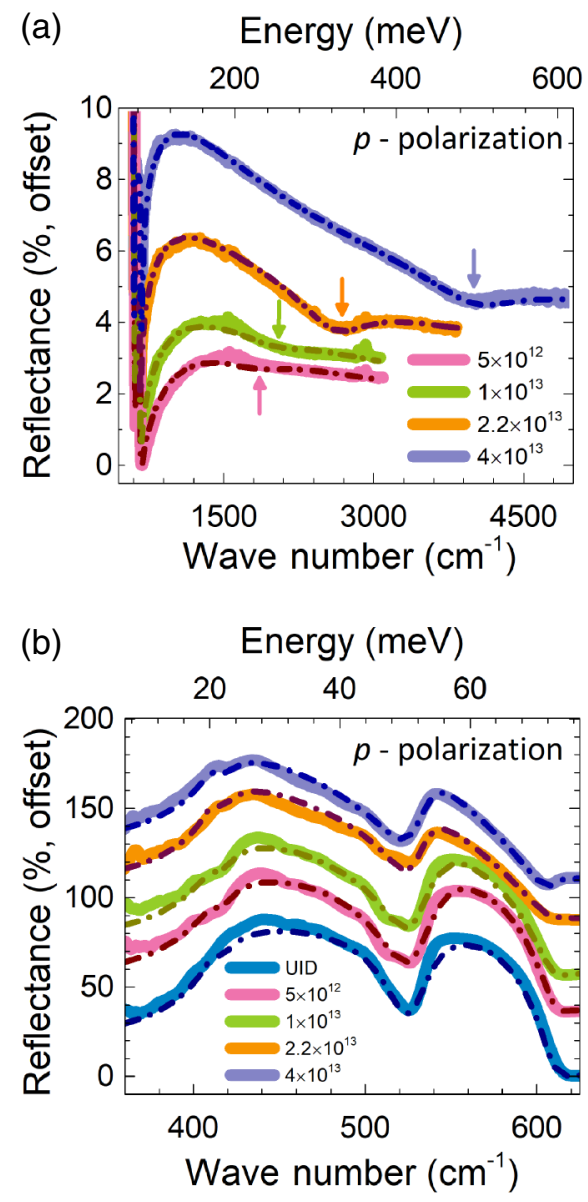

FIG. 3. IR reflectance results. (a) All doped samples in the study under $p$ polarization, offset for clarity. Arrows indicate the presence of the MSP. (b) Enlarged view of the reststrahlen band also under $p$ polarization, offset for clarity. The best fit for each spectra is overlaid on the experimental data in all the figures (dashed lines). Legends show the values of $n_{2 \mathrm{D}}$ extracted from reflectance experiments in units of $\mathrm{cm}^{-2}$ (see Ref. [24]).

angle of incidence of the light with respect to the normal to the plane of the QWs.

\section{RESULTS AND DISCUSSION}

The room-temperature $45^{\circ}$ IR reflectance spectra of the samples presented in this work are shown in Fig. 3(a) for $p$ polarization of the incident light. In all the spectra, the high-energy side of the reststrahlen band is seen at approximately $600 \mathrm{~cm}^{-1}$. The bump at approximately $1500 \mathrm{~cm}^{-1}$ is mostly due to the collective interaction of the free electrons with the light along the QW planes. A reflectance dip is observed - especially in the highly doped samples - that is not present in the spectra taken under $s$ polarization, fulfilling the polarization selection rule for
MSP resonance. Its presence in the samples with the lowest $2 \mathrm{D}$ electron concentration is also confirmed below with absorption spectroscopy.

Figure 3(b) shows the reflectance spectra at $45^{\circ}$ under $p$ polarization across the reststrahlen band. The main features apparent in all the spectra in the figure are also present in the spectrum corresponding to the undoped reference sample and is therefore explained by the interaction of the light with the phonon modes of doped $\mathrm{ZnO}$, undoped $\mathrm{ZnO}$, and $\mathrm{Mg}_{x} \mathrm{Zn}_{1-x} \mathrm{O}$. Note that the frequencies of interest for this work lie relatively close to the reststrahlen band, which spans from 409 to $590 \mathrm{~cm}^{-1}$ in $\mathrm{ZnO}$, i.e., from the $E_{1}(\mathrm{TO})$ to the $E_{1}(\mathrm{LO})$ frequencies in the measurement geometry employed here (see Sec. II and [24]). The reflectance valley around $525 \mathrm{~cm}^{-1}$ is due to the overlap between the reststrahlen bands corresponding to the two phonon modes in $\mathrm{Mg}_{x} \mathrm{Zn}_{1-x} \mathrm{O}$ when $\boldsymbol{E} \perp c$ [25]. The only effect electrons have in these spectra is that of their interaction with the inplane component of the electric field of the light, resulting in a tilt of the reflectance spectra which, in relative terms, becomes more intense at low-energy end of the reststrahlen band with increasing electron concentration.

Our use of a semiclassical approach with an anisotropic dielectric function to represent the interaction of light with in-plane free electrons and off-plane MSP reproduces very well the reflectance spectra of the doped sample under both $s$ and $p$ polarizations, including the frequency and lineshape of the ISB transitions [Fig. 3(a)]. The obtained values of the plasma frequency, $\omega_{p}$, and $n_{2 \mathrm{D}}$ are summarized in [24].

Note that for sample $D$ we obtain $n_{2 \mathrm{D}}=4 \times 10^{13} \mathrm{~cm}^{-2}$. Considering a monolayer of $m$-plane $\mathrm{ZnO}$ is approximately $0.21-\mathrm{nm}$ thick, each monolayer of $\mathrm{ZnO}$ would then contribute approximately $2 \times 10^{12}$ electrons $\mathrm{cm}^{-2}$ to the total two-dimensional electron density in the QW. This is to be compared to the case of GaAs, where the large twodimensional electron densities reported in ultrastrong coupling studies yield a contribution that approximately equals $3 \times 10^{11}$ electrons $\mathrm{cm}^{-2}$ per monolayer [31], i.e., roughly one order of magnitude smaller than in $\mathrm{ZnO}$.

Figure 4(a) shows the room-temperature absorbance of sample $C$ in a multipass waveguide configuration for $p$ and $s$ polarizations. In both cases, the absorbance increases for low energies due to absorption by free electrons: note in this configuration the light travels along $6 \mathrm{~mm}$ of material and, therefore, the samples are essentially opaque for wave numbers of the light below approximately $1000 \mathrm{~cm}^{-1}$. There is an outstanding feature around $2700 \mathrm{~cm}^{-1}(335 \mathrm{meV})$ which only appears in the spectrum measured under $p$ polarization, fulfilling the selection rule for MSP resonances. The MSP resonance can be better visualized if the ratio of the absorbance spectra taken at $p$ and $s$ polarizations is plotted [Fig. 4(b)], which allows measurement of the FWHM and verification that only one absorption peak is present (in agreement with 
(a)

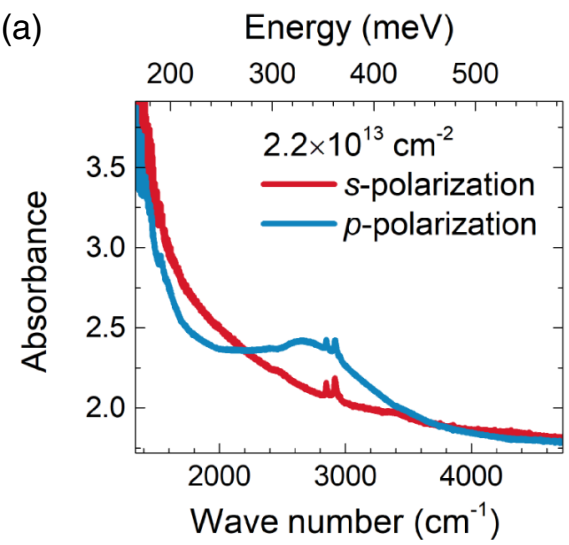

(b)

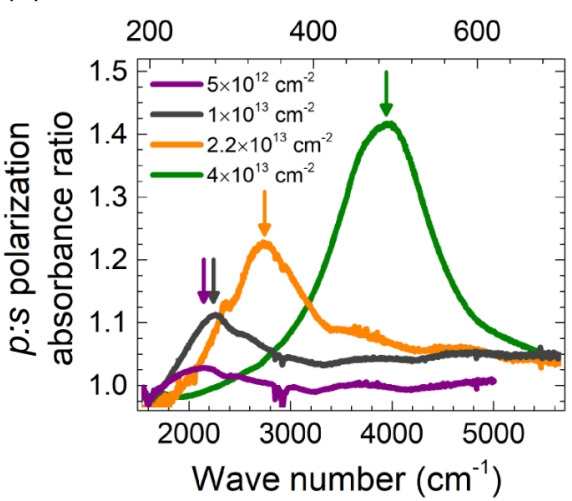

FIG. 4. MSP absorption. (a) Absorbance spectra of sample $C$ under $p$ and $s$ polarization of the incident light in a $45^{\circ}$ multipass waveguide configuration. (b) $p: s$ polarization absorbance ratio for all the doped samples in the study. Legends show the experimental values of $n_{2 \mathrm{D}}$.

what is expected from a MSP resonance). These peaks shift to higher energies as the $2 \mathrm{D}$ electron concentration is increased. Note that the peak energies correspond to what is observed in the reflectance experiments, and also that, consistently with the calculations of the MSP absorption coefficient, the MSP peak height increases with the electron concentration in the QW [32]. The FWHM of the measured MSP lies in the range of $800 \mathrm{~cm}^{-1}$, i.e., from $\gamma_{\text {ISBT }} / \tilde{\omega} \approx 0.38$ in the samples with the lowest $n_{2 \mathrm{D}}$ to $\gamma_{\text {ISBT }} / \tilde{\omega} \approx 0.28$ in the samples with the largest $n_{2 \mathrm{D}}$, where $\tilde{\omega}$ is the observed MSP peak frequency. This is similar to what was obtained by Belmoubarik et al. [14] in ISB transition photocurrent experiments at $18 \mathrm{~K}$ on $c$ plane $\mathrm{ZnO}$ QWs $\left(\gamma_{\mathrm{ISBT}} \approx 800 \mathrm{~cm}^{-1}, \gamma_{\mathrm{ISBT}} / \tilde{\omega} \approx 0.3\right)$, and lower than the values obtained by Zhao et al. [15] in room-temperature absorption experiments, also in $c$-plane ZnO QWs $\left(\gamma_{\mathrm{ISBT}} \approx 1250-1450 \mathrm{~cm}^{-1}, \gamma_{\mathrm{ISBT}} / \tilde{\omega} \approx 0.4\right)$.

The $\omega$ vs $n_{2 \mathrm{D}}$ dispersion relation of the MSP transitions, calculated from the absorption spectra of $\mathrm{ZnO}$ considering only the off-plane component of the dielectric function [see Sec. II and Eqs. (2a) and (8)], is now compared to the experimental data. Figure 5 (top) shows the spectral intensity of the MSP resonances together with the experimental results for the MSP peak energy extracted from Figs. 3(a) and 4(b) plotted against $n_{2 \mathrm{D}}$. The experimental data agrees excellently with the MSP model, where due to dipole-dipole interaction the oscillator strength is transferred from the lower- to the higherenergy transition in the QW as the Fermi energy moves towards and above $e_{2}$ [Fig. 1(b)]. Indeed, in the most heavily doped sample an independent transition model yields two absorption peaks with comparable intensities and at lower energies than the observed peak (see [24]), as opposed to the experiment. Our data show that when the depolarization-shifted independent transitions become resonant, the system becomes transparent for the transitions between adjacent levels, in agreement with the results in Li and Ning [33].

Moreover, the model employed here predicts that the intensity of the observed MSP increases with the 2DEG density, in agreement with the absorption curves in Fig. 4. This is also in agreement with the paper by Załużny [34] where it is found that for samples where the Fermi energy is above the first excited level of the QW, there are two resonances: one that shifts to higher energies and whose intensity increases with electron concentration, and another one whose energy and intensity decrease with increasing electron concentration. A more recent theory paper by Alpeggiani and Andreani [35] agrees with these observations as well. Also, note the unusual regime reached at these very dense 2DEGs where the depolarization-shifted MSP transition energy is up to three times the energy of the fundamental ISB transition for the most heavily doped sample.

The off-plane component of the dielectric function includes both the terms accounting for the MSP and the interaction of the light with the phonons. The model, therefore, predicts a coupling between these two oscillators. As $n_{2 \mathrm{D}}$ is increased, the LO-phonon resonance moves across the reststrahlen band towards $\omega_{\text {TO }}$ as a result of coupling (Fig. 5, bottom). This and the MSP resonances have similar intensities in the range of $2 \mathrm{D}$ electron concentrations from $10^{12}$ to $10^{13} \mathrm{~cm}^{-2}$, i.e., similar to the $n_{2 \mathrm{D}}$ ranges presented here. However, as a result of the large contribution of the $\mathrm{ZnO}$ substrate to the reflectance spectra together with the large broadening of the MSP, the lower branch of the dispersion relation (Fig. 5, bottom) is not observed in these samples. The broadening of the MSP also outshines the impact that the coupling with phonons is predicted to have on its frequency peak: approximately $40-\mathrm{cm}^{-1}$ MSP peak frequency shift vs approximately $800-\mathrm{cm}^{-1}$ MSP broadening. Its observation would thus require a more sophisticated system akin to the ISB polaron laser reported by Liu et al. [36], probably the first clear and unambiguous demonstration of an ISB polaron. In their work, a three-level GaAs/(Al,Ga)As intersubband 


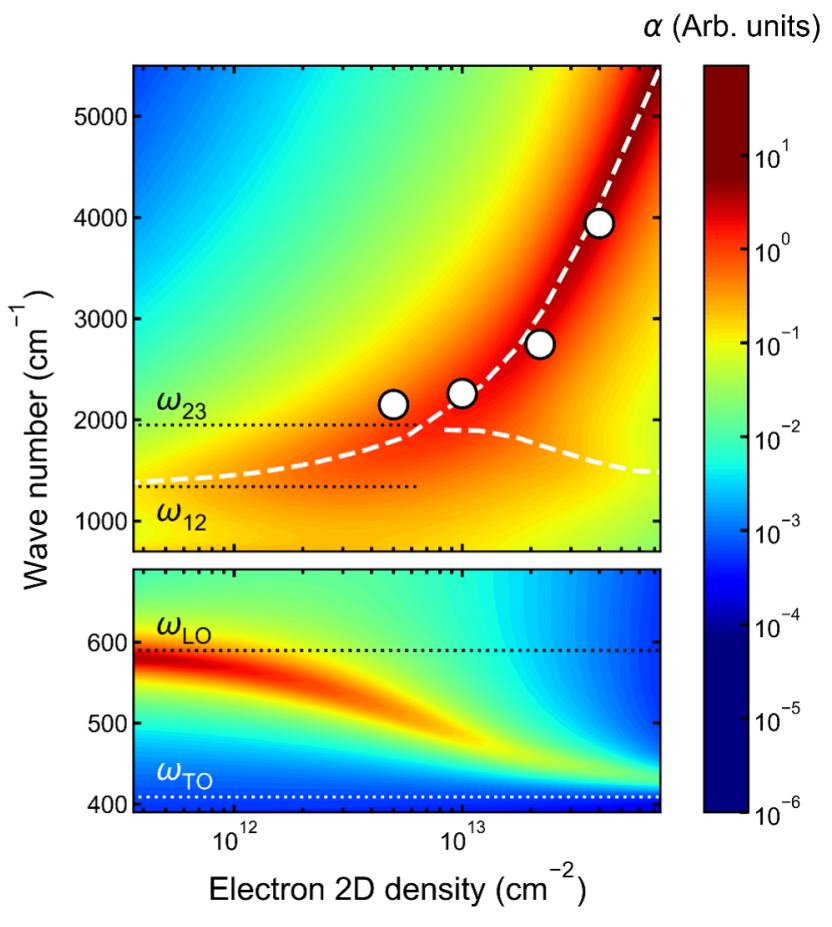

FIG. 5. MSP dispersion relation. $\omega$ to $n_{2 \mathrm{D}}$ dispersion relation calculated from Eq. (8). (Top) MSP resonance calculated expressing $\varepsilon_{z}$ as a sum of Lorentzian resonators as in the lefthand side of Eq. (3). The dotted horizontal lines indicate the original energies of the $e_{1} \rightarrow e_{2}$ and $e_{2} \rightarrow e_{3}$ transitions calculated from the self-consistent solution of the Schrödinger and Poisson equations. Dashed white lines indicate the maxima on the absorption spectra. Note how when the MSP reaches $\omega_{23}$ the $e_{1} \rightarrow e_{2}$ transition gradually disappears (bottom). Evolution of the phonon resonance with electron density due to the MSP-phonon coupling. Horizontal lines indicate the $E_{1}(\mathrm{LO})$ and $E_{1}(\mathrm{TO})$ frequencies from the $\mathrm{ZnO} \mathrm{QW}$.

laser was demonstrated at a temperature of $80 \mathrm{~K}$ where the Stokes Raman shift (i.e., the difference between the pump and lasing energies) was designed to be around the GaAs LO-phonon energy. It was found that, instead of having lasing action only when the Stokes Raman shift was equal to the LO-phonon resonance, the device would lase when the Stokes Raman shift was equal to the resonances of a dielectric function analogous to the (off-plane) one employed here. It can be foreseen that the same effect would be observed in a hypothetical $\mathrm{ZnO} / \mathrm{Mg}_{x} \mathrm{Zn}_{1-x} \mathrm{O}$ three-level laser designed with the Stokes Raman shift around the LO-phonon energy of $\mathrm{ZnO}$. In the $\mathrm{ZnO}$ case, though, the ISB polaronic gap for the Stokes Raman shift would be larger than $14 \mathrm{meV}$ (as opposed to approximately $6 \mathrm{meV}$ in the paper by Liu et al.) if the transition from the second to the first level of the three-level system is tuned to $\sqrt{\omega_{\mathrm{LO}}^{2}-\omega_{p}^{2}}$. This difference in the width of the ISB polaronic band gap between $\mathrm{GaAs}$ and $\mathrm{ZnO}$ would mainly be due to the larger difference between $\varepsilon_{\infty}$ and $\varepsilon(0)$ in $\mathrm{ZnO}$.

\section{CONCLUSIONS}

ISB transitions are demonstrated in $m$-plane homoepitaxial $\mathrm{ZnO} / \mathrm{Mg}_{x} \mathrm{Zn}_{1-x} \mathrm{O}$ MQWs with $2 \mathrm{D}$ electron densities in the QWs as high as $4 \times 10^{13} \mathrm{~cm}^{-2}$. It is found that the simultaneously occurring fundamental and excited-state ISB transitions couple into a single resonance, the multisubband plasmon, whose frequency is up to three times that of the fundamental transition. By means of a dielectric function anisotropic with respect to the growth plane of the crystal, the reflectance spectra are analyzed and serve to accurately quantify the actual electron concentrations in the QWs and to determine the $\omega$ to $n_{2 \mathrm{D}}$ dispersion relation of the MSP resonance. The results shown illustrate the potential of $m$-plane $\mathrm{ZnO} / \mathrm{Mg}_{x} \mathrm{Zn}_{1-x} \mathrm{O} \mathrm{QWs}$ for optoelectronic applications in the IR, which can be extended to the $\mathrm{THz}$ range by an appropriate design of the QW. The implications of these high $2 \mathrm{D}$ electron densities and the potential of $\mathrm{ZnO}$ to explore ISB-phonon coupling phenomena are discussed.

\section{ACKNOWLEDGMENTS}

We would like to thank Dr. Guzman for his help with the experimental setup and Professor B. Vinter for the critical reading of the manuscript. Part of the calculations performed here used the Aestimo solver [37]. This work is funded by the Spanish Ministry of Economy and Competitiveness (MINECO) through Projects TEC201460173-C2-2 and TEC2017-85912-C2-1-R, and from the European Union's Horizon 2020 Research and Innovation Program under Grant Agreement No. 665107 (project ZOTERAC). JTA holds a Predoctoral Contract from the Universidad Politécnica de Madrid.

[1] A. Tsukazaki, A. Ohtomo, T. Kita, Y. Ohno, H. Ohno, and M. Kawasaki, Quantum Hall effect in polar oxide heterostructures, Science 315, 1388 (2007).

[2] A. Ohtomo and H. Y. Hwang, A high-mobility electron gas at the $\mathrm{LaAlO}_{3} / \mathrm{SrTiO}_{3}$ heterointerface, Nature 427, 423 (2004).

[3] A. Tsukazaki, S. Akasaka, K. Nakahara, Y. Ohno, H. Ohno, D. Maryenko, A. Ohtomo, and M. Kawasaki, Observation of the fractional quantum Hall effect in an oxide, Nat. Mater. 9, 889 (2010).

[4] J. Falson, Y. Kozuka, M. Uchida, J. H. Smet, T.-H. Arima, A. Tsukazaki, and M. Kawasaki, $\mathrm{MgZnO} / \mathrm{ZnO}$ heterostructures with electron mobility exceeding $1 \times 10^{6} \mathrm{~cm}^{2} / \mathrm{Vs}$, Sci. Rep. 6, 26598 (2016).

[5] J. Falson, D. Maryenko, B. Friess, D. Zhang, Y. Kozuka, A. Tsukazaki, J. H. Smet, and M. Kawasaki, Evendenominator fractional quantum Hall physics in $\mathrm{ZnO}$, Nat. Phys. 11, 347 (2015).

[6] W. S. Baer, Faraday rotation in $\mathrm{ZnO}$ : determination of the electron effective mass, Phys. Rev. 154, 785 (1967). 
[7] S. Adachi, GaAs, AlAs, and $\mathrm{Al}_{x} \mathrm{Ga}_{1-x} \mathrm{As}$ : Material parameters for use in research, J. Appl. Phys. 58, R1 (1985).

[8] J. T. Devreese, in Encyclopedia of Applied Physics (WileyVCH Publishers, Inc, Weinheim, 1996), p. 383.

[9] K. J. Button, D. R. Cohn, M. von Ortenbert, B. Lax, E. Mollwo, and R. Helbig, Zeeman Splitting of Anomalous Shallow Bound States in ZnO, Phys. Rev. Lett. 28, 1637 (1972).

[10] S. J. Allen, Jr, D. C. Tsui, and B. Vinter, On the absorption of infrared radiation by electrons in semiconductor inversion layers, Solid State Commun. 20, 425 (1976).

[11] T. Ando, A. B. Fowler, and F. Stern, Electronic properties of two-dimensional systems, Rev. Mod. Phys. 54, 437 (1982).

[12] S. De Liberato and C. Ciuti, Quantum theory of intersubband polarons, Phys. Rev. B 85, 125302 (2012).

[13] N. Le Biavan, M. Hugues, M. Montes Bajo, J. TamayoArriola, A. Jollivet, D. Lefebvre, Y. Cordier, B. Vinter, F.-H. Julien, A. Hierro, and J.-M. Chauveau, Homoepitaxy of non-polar $\mathrm{ZnO} /(\mathrm{Zn}, \mathrm{Mg}) \mathrm{O}$ multi-quantum wells: From a precise growth control to the observation of intersubband transitions, Appl. Phys. Lett. 111, 231903 (2017).

[14] M. Belmoubarik, K Ohtani, and H Ohno, Intersubband transitions in $\mathrm{ZnO}$ multiple quantum wells, Appl. Phys. Lett. 92, 191906 (2008).

[15] K. Zhao, G. Chen, B. S. Li, and A. Shen, Mid-infrared intersubband absorptions in $\mathrm{ZnO} / \mathrm{ZnMgO}$ multiple quantum wells, Appl. Phys. Lett. 104, 212104 (2014).

[16] Ü. Özgür, Y. I. Alivov, C. Liu, A. Teke, M. A. Reshchikov, S. Doğan, V. Avrutin, S.- J. Cho, and H. Morkoç, A comprehensive review of $\mathrm{ZnO}$ materials and devices, J. Appl. Phys. 98, 041301 (2005).

[17] M. Beeler, E. Trichas, and E. Monroy, III-nitride semiconductors for intersubband optoelectronics: A review, Semicond. Sci. Technol. 28, 074022 (2013).

[18] P. Waltereit, O. Brandt, A. Trampert, H. T. Grahn, J. Menniger, M. Ramsteiner, M. Reiche, and K. H. Ploog, Nitride semiconductors free of electrostatic fields for efficient white light-emitting diodes, Nature 406, 865 (2000).

[19] S. Nakamura, The roles of structural imperfections in InGaN-based blue light-emitting diodes and laser diodes, Science 281, 956 (1998).

[20] D. Taïnoff, M. Al-Khalfioui, C. Deparis, B. Vinter, M. Teisseire, C. Morhain, and J.- M. Chauveau, Residual and nitrogen doping of homoepitaxial nonpolar m-plane $\mathrm{ZnO}$ films grown by molecular beam epitaxy, Appl. Phys. Lett. 98, 131915 (2011).

[21] S. Kalusniak, S. Sadofev, and F. Henneberger, ZnO as a Tunable Metal: New Types of Surface Plasmon Polaritons, Phys. Rev. Lett. 112, 137401 (2014).

[22] H. Yin, J. Chen, Y. Wang, J. Wang, and H. Guo, Composition dependent band offsets of $\mathrm{ZnO}$ and its ternary alloys, Sci. Rep. 7, 41567 (2017).

[23] M. D. Neumann, N. Esser, J.-M. Chauveau, R. Goldhahn, and M. Feneberg, Inversion of absorption anisotropy and bowing of crystal field splitting in wurtzite $\mathrm{MgZnO}$, Appl. Phys. Lett. 108, 221105 (2016).

[24] See Supplemental Material at http://link.aps.org/supple mental/10.1103/PhysRevApplied.10.024005 for the parameters employed in the dielectric function calculation, the geometry of the transmittance and reflectance experiments, a note on the detection limit for electron concentration by reflectance, and a comparison of multiple ISB transition models.

[25] C. Bundesmann, A. Rahm, M. Lorenz, M. Grundmann, and $\mathrm{M}$. Schubert, Infrared optical properties of $\mathrm{Mg}_{x} \mathrm{Zn}_{1-x} \mathrm{O}$ thin films $(0 \leq x \leq 1)$ : Long-wavelength optical phonons and dielectric constants, J. Appl. Phys. 99, 113504 (2006).

[26] W. P. Chen, Y. J. Chen, and E. Burnstein, The interface EM modes of a "surface quantized" plasma layer on a semiconductor surface, Surf. Sci. 58, 263 (1976).

[27] P. Yu and M. Cardona, Fundamentals of Semiconductors. Physics and Materials Properties (Springer-Verlag, Berlin Heidelberg, 2010).

[28] B. Askenazi, A. Vasanelli, A. Delteil, Y. Todorov, L. C. Andreani, G. Beaudoin, I. Sagnes, and C. Sirtori, Ultrastrong light-matter coupling for designer Reststrahlen band, New J. Phys. 16, 043029 (2014).

[29] R. Steed, Transfer matrix theory for a type of uniaxial layers: Starting from basic electromagnetism, Available at https://www.researchgate.net/publication/236491437 (2013).

[30] M. Załużny and C. Nalewajko, Coupling of infrared radiation to intersubband transitions in multiple quantum wells: The Effective Medium Approach, Phys. Rev. B 59, 13043 (1999).

[31] T. Laurent, Y. Todorov, A. Vasanelli, A. Delteil, C. Sirtori, I. Sagnes, and G. Beaudoin, Superradiant Emission from a Collective Excitation in a Semiconductor, Phys. Rev. Lett. 115, 187402 (2015).

[32] A. Delteil, A. Vasanelli, Y. Todorov, C. Feulliet Palma, M. Renaudat St-Jean, G. Beaudoin, I. Sagnes, and C. Sirtori, Charge-Induced Coherence between Intersubband Plasmons in a Quantum Structure, Phys. Rev. Lett. 109, 246808 (2012).

[33] J. Li and C. Z. Ning, Induced Transparency by Intersubband Plasmon Coupling in a Quantum Well, Phys. Rev. Lett. 93, 087402 (2004).

[34] M. Załużny, On the influence of the depolarization field on the intersubband absorption in thin semiconductor films, Phys. Status Solidi B 123, K57 (1984).

[35] F. Alpeggiani and L. C. Andreani, Semiclassical theory of multisubband plasmons: Nonlocal electrodynamics and radiative effects, Phys. Rev. B 90, 115311 (2014).

[36] H. C. Liu, C. Y. Song, Z. R. Wasilewski, A. J. SpringThorpe, J. C. Cao, C. Dharma-wardana, G. C. Aers, D. J. Lockwood, and J. A. Gupta, Coupled Electron-Phonon Modes in Optically Pumped Resonant Intersubband Lasers, Phys. Rev. Lett. 90, 077402 (2003).

[37] The solver can be found at http://www.aestimosolver.org/ 